\title{
EcoLogic: um jogo de estratégia para o desenvolvimento do pensamento computacional e da consciência ambiental
}

\author{
Fernanda Gabriela Pires ${ }^{1,2}$, Rafaela Ferreira ${ }^{1}$, Marcos Guibson Silva ${ }^{1}$, Jean \\ Batista $^{1}$, Fabrizio Franzoia ${ }^{1}$, Rosiane de Freitas ${ }^{2}$ \\ ${ }^{1}$ Licenciatura em Computação, Laboratório de Tecnologias Educacionais - \\ Universidade do Estado do Amazonas (UEA) \\ Av. Darcy Vargas, 1.200- 69050-020 - Manaus - AM - Brazil \\ ${ }^{2}$ Instituto de Computação - Universidade Federal do Amazonas (UFAM) \\ Manaus, AM - Brasil \\ \{fpires, rmf.lic16, mgss.lic17, jsmb.lic16, fhf.lic17\}@uea.edu.br, \\ rosiane@icomp.ufam.edu.br
}

\begin{abstract}
This article deals with the proposal of an educational game, which aims to develop Computational Thinking by understanding and caring for the environment in which one lives, in order to generate environmental and ecological awareness. EcoLogic is a digital puzzle and strategy game, whose mission is to explore the environment by solving logical problems. The theme addressed is sustainability through selective garbage collection. It is anchored in the four pillars of Computational Thinking and uses Constructionism as the principle of action. The game was developed for children from 8 years of age, with application recommended for use in schools, as a pedagogical support, as an entertainment tool for children, youth and adults.
\end{abstract}

Resumo. O presente artigo trata da proposta de um jogo educacional, que visa desenvolver o Pensamento Computacional através do entendimento e cuidados com o ambiente em que se vive, com intuito de gerar consciência ambiental e ecológica. EcoLogic é um jogo digital de ação e estratégia em formato de puzzle, cuja missão do jogador consiste em explorar o ambiente resolvendo problemas lógicos. O tema tratado é a sustentabilidade através da coleta seletiva de lixo. Está ancorado nos quatro pilares do Pensamento Computacional e usa o Construcionismo como princípio de ação. O jogo foi desenvolvido para crianças a partir de oito anos, com aplicação recomendada tanto para uso em escolas, como apoio pedagógico, como uma ferramenta de entretenimento por crianças, jovens e adultos.

\section{Introdução}

Muito vem se discutindo sobre o desenvolvimento do Pensamento Computacional (PC) como uma habilidade essencial para a resolução de problemas quaisquer em situações do dia-a-dia ao longo da vida, na forma de enxergar os elementos importantes de decisão as variáveis - e estabelecer conexões entre os mesmos. Tais discussões ganharam forma através do discurso de Jeannette Wing (2006) que define PC como uma habilidade fundamental para qualquer pessoa e não somente um Cientista da Computação, deixando claro que o pensamento crítico deve ser associado a elementos da computação, e que PC seria uma ferramenta para formalização na resolução de problemas. 
VII Congresso Brasileiro de Informática na Educação (CBIE 2018)

Anais dos Workshops do VII Congresso Brasileiro de Informática na Educação (WCBIE 2018)

É fato que estratégias de resolução de problemas são requeridas em todos os campos da vida, inclusive em simulações apresentadas na forma de entretenimento, como em jogos. Os jogos vêm sendo defendidos como alternativas lúdicas no processo de aprendizagem (Alves, 2015; Dichev \& Dicheva, 2017), por proporcionarem interação e engajamento por meio do envolvimento entre o jogador e a ação. A utilização de jogos em processos educacionais ainda suscita muitas discussões, pois criar um jogo com propósitos educativos exige muito mais do que o necessário a jogos comerciais puramente de entretenimento, que ainda assim já possuem um processo complexo de desenvolvimento.

Ambiente e sustentabilidade são temas complexos nos dias atuais, onde a conscientização sobre os cuidados com o ambiente não tem se mostrado uma tarefa fácil ao redor do mundo, como mostram as discussões que acompanham os grandes tratados mundiais em nome do clima, entre elas as conferências definidas a partir do Clube de Roma (Fogel, 1994; Meadows \& Randers, 2012).O desenvolvimento do Pensamento Computacional é essencial para a resolução de problemas e atingir a sustentabilidade é um fator muito desejado atualmente, sobretudo no que tange ao tratamento do lixo e aos cuidados que podem ser realizados pela população em geral. Considerando este contexto, propõe-se o desenvolvimento de um jogo eletrônico que usa como base os quatro pilares do Pensamento Computacional na construção de estratégias para a resolução de problemas. Tal jogo estabelece uma relação entre estratégias lógicas através de percursos em labirintos e a coleta seletiva de lixo.

O restante deste artigo está organizado como segue. Na Seção 2 são apresentados os trabalhos relacionados. Na Seção 3, são apresentados os fundamentos teóricos sobre Pensamento Computacional, jogos e gamificação em processos de aprendizagem, bem como a temática de conscientização ambiental. Na Seção 4 é apresentado o jogo educacional EcoLogic proposto. Resultados são citados na Seção 5 e, por fim, na Seção 6 são feitas as considerações finais e indicados os trabalhos futuros.

\section{Trabalhos Relacionados}

Silva \& Passerino (2007) apresentam um jogo de propósito sério com as temáticas "sustentabilidade, ambiente e interdisciplinaridade”, intitulado de "A fazenda”. O jogo tem por objetivo utilizar a simulação de um ambiente de gerenciamento de uma fazenda, se mostrando como uma alternativa lúdica no auxílio ao processo de aprendizagem. Os autores ressaltam a carência de ferramentas que atendam tais necessidades no Brasil.

Farias, Andrade, \& Alencar (2015) discutem sobre Pensamento Computacional em sala de aula, seus desafios, possibilidades e a formação docente. Os autores analisam a forma como o PC vem sendo trabalhado, suas possibilidades e limitações, e o resultado disso foi um compêndio que contemplou aspectos como infraestrutura, acesso à Internet e atividades escolares. Os resultados apontaram para um corpo docente despreparado para atuar com a prática do Pensamento Computacional.

Qian \& Clark (2016) apresentam uma revisão sistemática da literatura sobre aprendizagem baseada em jogos (do inglês, Game-Based Learning GBL), bem como teorias de aprendizagem e habilidades do Século XXI. Segundo a revisão de literatura realizada por eles, GBL pode ser eficaz no que tange a simplificação do processo de desenvolvimento dessas habilidades, e frente a isto é mostrado o exemplo do atual 
VII Congresso Brasileiro de Informática na Educação (CBIE 2018)

Anais dos Workshops do VII Congresso Brasileiro de Informática na Educação (WCBIE 2018)

currículo escolar de Hong Kong, o qual enfatiza a importância da construção da criatividade dos alunos, e assim, os professores são incentivados a promover inovação.

Diferente das pesquisas anteriores, o presente trabalho integra a importância da sustentabilidade e do Pensamento Computacional através do potencial apresentado pela aprendizagem baseada em jogos. O serious game intitulado Ecologic, possui temática de meio ambiente e instiga a resolução de problemas a partir de estratégias baseadas nos 4 pilares do PC.

\section{Fundamentos Teóricos}

\subsection{Pensamento Computacional}

Wing (2006) considera o Pensamento Computacional (PC) uma habilidade fundamental para todos, que permite a resolução de problemas de forma analítica e diz respeito a forma como as pessoas veem e estruturam o pensamento. Dessa forma, o pensamento computacional, bem como a leitura, a escrita e a aritmética, deve fazer parte da capacidade analítica de todas as pessoas, desde a infância. Em 2010 (Smith, Sutcliffe, \& Sandvik, 2014) definiram os processos de pensamento utilizados na resolução de um problema, cuja representação permite a execução por um agente de processamento de informações.

PC é uma habilidade que envolve a resolução de problemas através de conceitos computacionais, dentre estes, estão os 04 (quatro) pilares fundamentais (BBC, 2018): Decomposição, Reconhecimento de Padrões, Abstração e Algoritmos. Ao se deparar com uma tarefa difícil, "pensar computacionalmente” é reestruturar o problema de forma que a solução seja mais facilmente encontrada. Parafraseando a forma como as máquinas chegam a soluções de problemas complexos, isso é possível através dos 4 pilares (Figura 1).

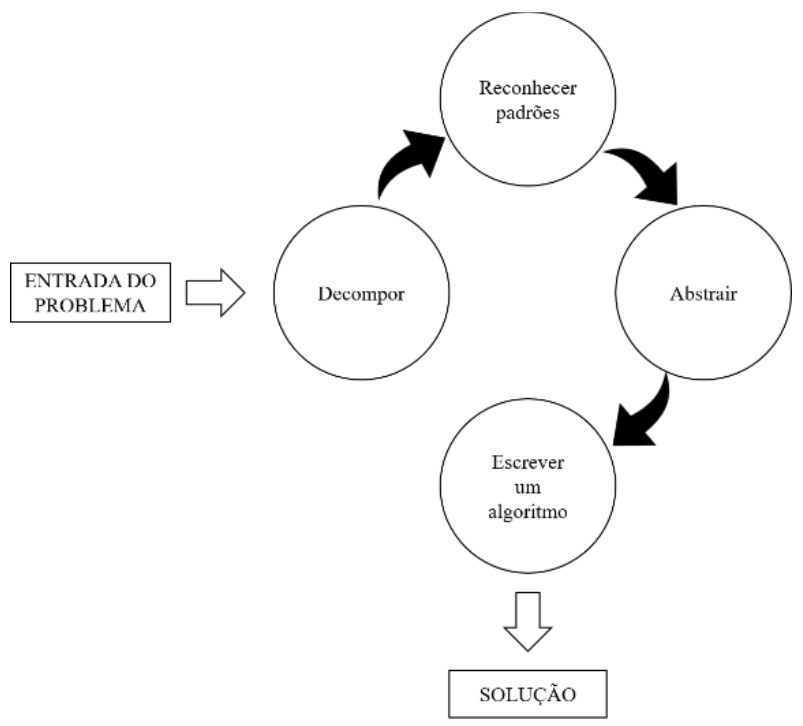

Figura 1. Processo de resolução de um problema, segundo os pilares do Pensamento Computacional (PC).

A primeira etapa é a Decomposição, quando ao receber um problema complexo, o indivíduo deve dividi-lo em partes menores e mais simples para facilitar a compreensão e otimizar o entendimento. Após decompor o problema, a segunda fase é o Reconhecimento de Padrões, onde se examina os problemas menores de forma a encontrar padrões entre eles, e tais padrões são úteis, pois, ao encontrar a solução para 
VII Congresso Brasileiro de Informática na Educação (CBIE 2018)

Anais dos Workshops do VII Congresso Brasileiro de Informática na Educação (WCBIE 2018)

um dos problemas pequenos, pode-se reutilizá-la para a resolução de outros problemas, ou seja, a solução é replicável. Quanto mais padrões foram encontrados, mais fácil e rápida se tornará a resolução de problemas.

A terceira etapa do processo é a Abstração, talvez este seja um dos mais importantes pilares, em que se identificam as características mais relevantes para solucionar o problema e se desconsideram detalhes de menor relevância, de maneira a formalizar a ideia geral do problema (BBC, 2018). A quarta e última etapa é a formalização dos Algoritmos capazes de serem aplicados com a finalidade de resolver o problema, após decompor, reconhecer padrões e abstrair, são determinadas regras para a resolução dos problemas. Um algoritmo é um conjunto de instruções que descreve os passos para solucionar um problema.

\subsection{Jogos e processos educacionais}

Ao analisar a importancia da disseminação de métodos e tecnicas capazes de auxiliar no desnvolvimento do Pensamento Computacional, se observou a utilização de jogos digitais no cenário educacional, e como tem se mostrado uma ferramenta com vistas a tornar a aprendizagem mais atraente e divertida (Savi \& Ulbricht, 2008). Sabe-se que as crianças de hoje nascem em meio a tecnologia e crescem utilizando jogos de computador, videogames e smartphones, reservando grande parcela do seu tempo a estas atividades. E conforme a neuroplasticidade, o cérebro tem a capacidade de criar novas conexões com base nos inputs recebidos, facilitando o processo de aprendizagem. Considerando isso, a familiaridade com as mecânica de jogos, surgem propostas de aplicação dos mesmos na educação, ou ainda a gamificação de processos com mesmo cunho (Dichev \& Dicheva, 2017).

Para que a aprendizagem por meio dos jogos seja efetiva é preciso levar em conta não apenas o aspecto de diversão dos jogos tradicionais, mas sim, os aspectos cognitivos, ou seja, no processo de desenvolvimento de um jogo educacional é importante pensar que habilidades e estratégias resultarão no aprimoramento de capacidades intelectuais (Gros, 2003). Geralmente, os jogos comerciais são construídos para proporcionar diversão e não com foco em aprendizagem, onde a constituição de um jogo educacional predispõe uma série de fatores que precisam ser avaliados. A constituição de um jogo educacional que de fato consiga atender aos requisitos lúdicos e ainda seja educacionalmente construtivo, é um desafio (Berg Marklund, 2013), para tanto precisa estar de acordo com as estruturas cognitivas humanas e ainda sim incorporar a carga cognitiva intrínseca ao conteúdo preferencialmente de forma implícita (Mayer, 2015) .

Quando implementados da maneira correta, os softwares educacionais possuem diversos benefícios, alguns deles são (Mitchell \& Savill-Smith, 2004, Hsiao, 2007):

- Efeito motivador: por serem atraentes e divertidos, os jogos costumam deixar o jogador mais relaxado, proporcionando dessa forma maior disposição para o aprendizado (Hsiao, 2007), além disso, os frequentes desafios despertam nas pessoas motivação para vencê-los.

- Aprendizagem facilitada: conteúdos considerados difíceis podem ser representados de maneira mais simples no cenário de um jogo, facilitando dessa forma, o aprendizado. Os jogos podem também estimular a aprendizagem por meio da tentativa e do erro (Mitchell \& Savill-Smith, 2004). 
VII Congresso Brasileiro de Informática na Educação (CBIE 2018)

Anais dos Workshops do VII Congresso Brasileiro de Informática na Educação (WCBIE 2018)

- Desenvolvimento de habilidades cognitivas: ao longo de um jogo, o usuário é constantemente encorajado a desenvolver estratégias, aspecto relevante para a resolução de problemas, memorização, raciocínio dedutivo (McFarlane, Sparrowhawk, \& Heald, 2002), insight e desenvolvimento de habilidades analíticas e computacionais (Mitchell \& Savill-Smith, 2004).

É importante observar que um importante papel desenvolvido pelos jogos é possibilitar a simulação do mundo real em ambientes digitais, permitindo vivenciar experiências sem de fato correr riscos, de forma engajadora em decorrência das mecânicas envolvidas.

\subsection{Ambiente e Sustentabilidade}

O surgimento do termo sustentabilidade foi oficialmente introduzido no encontro internacional The Word Conservation Strategy (Calvacanti, 1995), quando surgiu a definição do termo Desenvolvimento Sustentável em 1980, definindo como mundo ideal práticas capazes de satisfazer o presente sem influenciar ou comprometer 0 desenvolvimento das novas gerações (Siche, Agostinho, Ortega, \& Romeiro, 2007).

Apesar de que atualmente o termo Sustentabilidade é comumente associado a outros termos, Siche (Siche et al., 2007) refere-se à sustentabilidade como tudo que pode ser mantido, conservado ou suportado. Sachs (1989) descreve a sustentabilidade como um conceito dinâmico que toma como base as constantes necessidades advindas da sociedade. No contexto de sustentabilidade, encontra-se a educação ambiental, tendo como objetivo central auxiliar nas boas práticas que guiam a relação do homem com ambiente (Sauvé, 2005).

Em tempos em que as informações possuem um papel de extrema relevância para o contexto social, a educação ambiental juntamente com cidadania detém a grande capacidade de motivar e sensibilizar pessoas para transformar as diversas formas de participação da defesa da qualidade de vida (Jacobi, 2003). Deste modo, entende-se que a educação ambiental é condição necessária para que haja a uma modificação no quadro socioeconômico (Sauvé, 2005), por isso a proposta tem como objetivo a promoção de educação ambiental no que diz respeito a coleta seletiva através de um jogo eletrônico que de forma implícita, promove o desenvolvimento de estruturas do PC.

\section{EcoLogic: aplicativo móvel para educação ambiental}

EcoLogic é um jogo digital educacional de ação e estratégia em formato de puzzle, para plataformas móveis, com perspectiva top-down, que envolve mecânicas de estilos de jogos de ação e aventura, com aplicações de puzzle e possui personagens com estilos artísticos de RPG Maker. Procura introduzir noções de educação ambiental, relacionadas à coleta seletiva, na associação de tipos de lixos às suas respectivas lixeiras, e disseminar o pensamento computacional voltado para a resolução de problemas lógicos.

EcoLogic proporciona ao usuário conhecer o processo de reciclagem, apresentando as cores da coleta seletiva. O objetivo do jogador é recolher os diferentes materiais (lixos) espalhados no cenário e levá-los para a lixeira correspondente. As lixeiras estão dispostas em pontos estratégicos, que provoca ao jogador a necessidade de fazer uso do pensamento lógico para se esquivar dos diversos inimigos. Os inimigos são criaturas que vagam em meio ao lixo e dificultam o jogador a alcançar as lixeiras para o descarte correto dos materiais e causam danos quando colidem com a personagem. Os 
VII Congresso Brasileiro de Informática na Educação (CBIE 2018)

Anais dos Workshops do VII Congresso Brasileiro de Informática na Educação (WCBIE 2018)

cenários estão estruturados em forma de labirinto, proporcionando ao jogador a promoção do pensamento computacional, na resolução de problemas, para conseguir se esquivar dos inimigos e levar os materiais até as lixeiras.

A tabela abaixo (Tabela 1 ) estabelece uma relação entre os pilares do pensamento computacional, estruturas computacionais e a relação que se pretendo com o meio ambiente.

Tabela 1. Pensamento Computacional e o EcoLogic.

\begin{tabular}{|c|c|c|c|}
\hline $\begin{array}{c}\text { Pensamento } \\
\text { Computacional }\end{array}$ & EcoLogic & $\begin{array}{c}\text { Estruturas } \\
\text { Computacionais }\end{array}$ & Meio Ambiente \\
\hline Decomposição & $\begin{array}{l}\text { Setorização das áreas de ação } \\
\text { Localização de objetivos } \\
\text { Classificação de lixeiras e } \\
\text { inimigo }\end{array}$ & $\begin{array}{l}\text { Variáveis } \\
\text { Laços de repetição } \\
\text { Condicionais }\end{array}$ & $\begin{array}{l}\text { Relação entre o jogo e o } \\
\text { meio }\end{array}$ \\
\hline Padrão & $\begin{array}{l}\text { Organização espacial } \\
\text { Número de árvores } \\
\text { Formação de obstáculos } \\
\text { Localização de alvos }\end{array}$ & \multirow{3}{*}{$\begin{array}{l}\text { Disjunção } \\
\text { Conjunção } \\
\text { Repetição } \\
\text { Condicionais } \\
\text { Caminho mínimo } \\
\text { Laços de repetição }\end{array}$} & $\begin{array}{l}\text { Localização de padrão } \\
\text { Correlato a diferentes } \\
\text { ambientes }\end{array}$ \\
\hline Abstração & $\begin{array}{l}\text { Posição dos lixos e lixeiras } \\
\text { Movimentação dos inimigos } \\
\text { HUD de feedback } \\
\text { Corações (vidas) }\end{array}$ & & $\begin{array}{l}\text { Descarte do lixo na } \\
\text { respectiva lixeira } \\
\text { Poluição do ambiente } \\
\text { pelos inimigos }\end{array}$ \\
\hline Algoritmo & $\begin{array}{l}\text { Sequência de passos para: } \\
\text { - Contornar obstáculos } \\
\text { - Cumprir metas } \\
\text { - Contornar inimigos }\end{array}$ & & $\begin{array}{l}\text { Como proceder para } \\
\text { manter o ambiente bem } \\
\text { cuidado e promover } \\
\text { sustentabilidade } \\
\text { ambiental } \\
\end{array}$ \\
\hline
\end{tabular}

Para cumprir a tarefa proposta pelo jogo, o usuário precisará usar raciocínio lógico, a tela de ação foi dividida em uma grande matriz onde os elementos estão dispostos. Como obstáculos fixos foram colocadas várias árvores, algumas em sequência de barras, outras em sequências de "L". As lixeiras estão dispostas de forma aleatória pelo cenário, onde na primeira fase existe um único tipo de lixeira, e, então, o dever do jogador é simplesmente não se deixar alcançar pelos "monstros" dispostos pelo cenário. As lixeiras por vezes adquirem a mesma mecânica encontrada no famoso jogo Sokoban, ou seja, se não for levada para o local certo, fica uma "peça morta”.

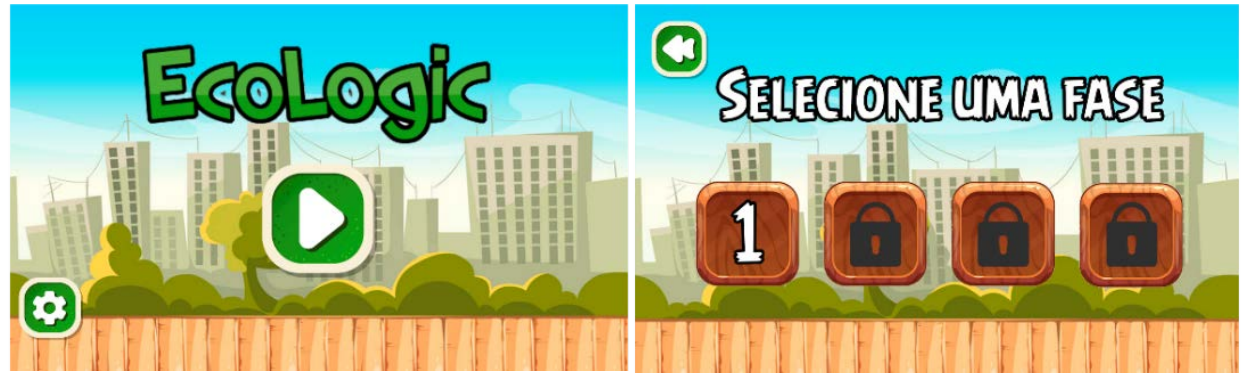

Figura 2. Tela Principal e Seleção de Fases.

Para fazer o descarte correto dos itens de lixo, o jogador precisa movimentar a personagem para tanto, faz uso dos comandos touch screen nas extremidades da tela - direita, esquerda, superior e inferior - é a personagem que levará o material para as lixeiras específicas. A visão top-down (Figura 3) e a movimentação da câmera que acompanha o jogador são algumas das características do EcoLogic. A vitória é condicionada a dois critérios, primeiro, o jogador precisa desviar dos inimigos 
VII Congresso Brasileiro de Informática na Educação (CBIE 2018)

Anais dos Workshops do VII Congresso Brasileiro de Informática na Educação (WCBIE 2018)

para não sofrer danos e acabar morrendo, segundo, é preciso fazer a coleta seletiva de todo material espalhado pelo cenário, só assim a porta que leva para o próximo nível é desbloqueada.

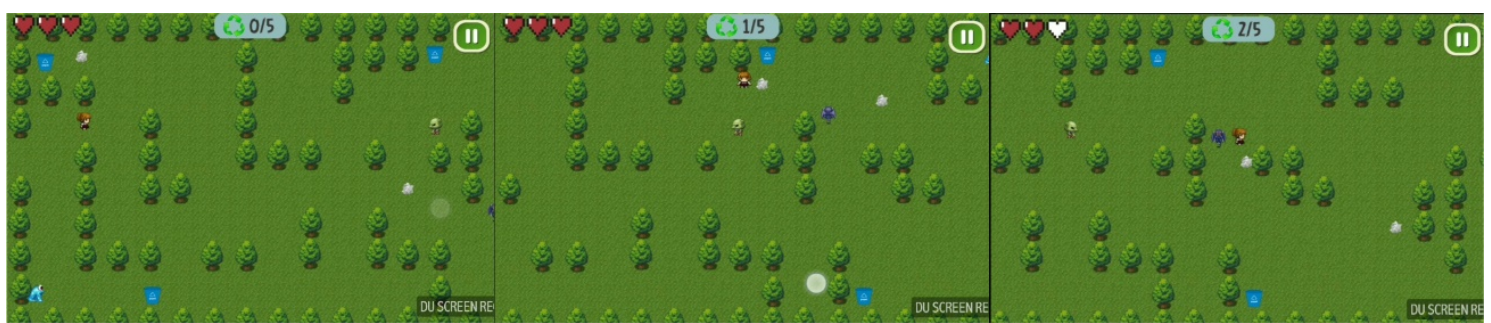

Figura 3. Tela de Gameplay com HUD's.

Tabela 2. Pensamento Computacional e o passo a passo do jogo.

\begin{tabular}{|ll|}
\hline $\begin{array}{c}\text { Pensamento } \\
\text { Computacional }\end{array}$ & \multicolumn{1}{c|}{ Gameplay } \\
\hline Decomposição & $\begin{array}{l}\text { Para movimentar-se pela tela, o usuário precisa decompor, esquadrinhar a tela } \\
\text { em busca de saídas para cumprir os seus objetivos, seja fugir dos vilões ou levar } \\
\text { o lixo à lixeira }\end{array}$ \\
\hline $\begin{array}{l}\text { Reconhecimento de } \\
\text { padrões }\end{array}$ & $\begin{array}{l}\text { Após o processo de separar por quadros a tela, o usuário começa ambientar-se } \\
\text { com os controles e os padrões de movimento e disposição dos obstáculos } \\
\text { definindo a existência de barreiras móveis e fixas a fim de montar estratégias de } \\
\text { movimento }\end{array}$ \\
\hline Abstração & $\begin{array}{l}\text { No processo de busca por objetivos, o usuário deve perceber que existem } \\
\text { possibilidades específicas para cumprir os dois objetivos gerais: fuga dos vilões e } \\
\text { levar o lixo à lixeira. Caso seu movimento aprisione uma peça, não existirá mais } \\
\text { a possibilidade de finalizar a fase com pontos completos }\end{array}$ \\
\hline Algoritmo & $\begin{array}{l}\text { O processo de traçar a melhor rota, sem perder vidas e cumprindo as metas } \\
\text { estabelecidas da coleta. }\end{array}$ \\
\hline
\end{tabular}

Todos os pilares defendidos como importantes para o desenvolvimento do Pensamento Computacional estão diluídos nas estruturas do jogo, que pretende engajar o jogador tanto com a sequência de passos a ser realizada quanto com a gamificação em si.

\subsection{Validação}

A avaliação de EcoLogic foi feita com a versão digital do game, a partir do método de avaliação de jogos educacionais EGameFlow (Fu, Su, \& Yu, 2009), uma adaptação do método GameFlow (Dias et al., 2015), porém, difere deste ao conter aspectos educacionais de jogos. Dada a importância da interação e avaliação do usuário com o jogo educacional, o método EGameFlow procura justamente uma escala de satisfação entre estes (usuário-jogo educacional), podendo auxiliar o game designer a compreender os pontos fortes e fracos do jogo em perspectiva do jogador (Tsuda, Sanches, Ferreira, Otsuka, \& Beder, 2014).

Tabela 3. Resultados dos testes de EGameFlow.

\begin{tabular}{lccccccc}
\hline Critérios & 0 & 1 & 2 & 3 & 4 & 5 & 6 \\
\hline Concentração & $5 \%$ & $5 \%$ & $15 \%$ & $20 \%$ & $25 \%$ & $15 \%$ & $15 \%$ \\
Desafios & $16 \%$ & $18 \%$ & $12 \%$ & $18 \%$ & $10 \%$ & $18 \%$ & $8 \%$ \\
Autonomia & $8,8 \%$ & $24,4 \%$ & $4,4 \%$ & $15,5 \%$ & $8,8 \%$ & $17,7 \%$ & $20 \%$ \\
Clareza dos objetivos & $0 \%$ & $0 \%$ & $4 \%$ & $16 \%$ & $24 \%$ & $16 \%$ & $40 \%$ \\
Feedback & $6,6 \%$ & $6,66 \%$ & $13,3 \%$ & $13,3 \%$ & $6,6 \%$ & $16,6 \%$ & $36,6 \%$ \\
Imersão & $8,5 \%$ & $37,1 \%$ & $5,7 \%$ & $14,2 \%$ & $20 \%$ & $5,71 \%$ & $8,5 \%$ \\
Interação Social & $53,3 \%$ & $26,6 \%$ & $0 \%$ & $6,6 \%$ & $6,6 \%$ & $0 \%$ & $6,6 \%$ \\
\hline
\end{tabular}


VII Congresso Brasileiro de Informática na Educação (CBIE 2018)

Anais dos Workshops do VII Congresso Brasileiro de Informática na Educação (WCBIE 2018)

A partir do EGameFlow, foram avaliados 7 critérios: Concentração, Desafios, Autonomia, Clareza dos Objetivos, Feedback, Imersão e Interação Social (Tsuda et al., 2014), contendo estes, vários itens que são analisados para chegar aos resultados dos testes Tabela 3.

Os testes foram realizados com estudantes de graduação em computação (Engenharia e Licenciatura) e contou com uma unidade amostral $\mathrm{N}=07$, entretanto, o jogo foi desenvolvido para crianças com faixa etária entre 08 e 12 anos, o que pode apresentar uma ameaça a validade. EcoLogic apresentou as melhores porcentagens (5 e 6) nos critérios de Clareza dos objetivos (56\%) e Feedback (53,32\%). Durante o jogo, muitos usuários demonstraram dificuldade em organizar os quebra cabeças apresentados de forma a criar as melhores estratégias para levar o lixo até as suas lixeiras, sobretudos nas sessões em que havia inimigos como sentinelas, o que aponta para a necessidade de redução no nível de dificuldade da primeira fase.

Quando perguntados sobre o jogo, os usuários entrevistados indicaram gostar da proposta, mas apontaram para a necessidade de melhorias no design, e como o jogo está em processo de desenvolvimento, as observações serão incorporadas ao próximo protótipo para testes, um processo cíclico até que o jogo fique pronto para uso comum.

\section{Resultados e discussões}

O propósito do desenvolvimento do jogo EcoLogic é o de fornecer uma ferramenta multidisciplinar, lúdica para promoção do desenvolvimento do Pensamento Computacional de maneira formal e não formal, ou seja, o jogo pode tanto ser jogado de forma independente por qualquer usuário, como pode ser usado em sala de aula de forma colaborativa com a presença de crianças e seu tutor.

As estruturas computacionais podem ser trabalhadas de forma a pedir que as crianças desenvolvam algoritmos no formato disponível no site code.org, com a missão Minecraft, em que o cenário é semelhante. O tutor pode exaltar as palavras chaves do tipo "Se" a personagem for pela direita "então", "Se" lixeira na localização "x”. Além de traçar as menores rotas possíveis para se alcançar um alvo ou fugir dos vilões, pode-se explorar trabalhos com planos cartesiano, senso de lateralidade, entre outros. O jogo pode ser simulado em uma lousa digital interativa, possibilitando uma participação de um maior número de pessoas que poderão traçar estratégias de forma colaborativa em grupos, além do engajamento pela competitividade.

Diante das discussões da inserção do Pensamento Computacional como componente curricular nas escolas, se faz necessário o desenvolvimento de métodos, técnicas e ferramentas que possam ser utilizados em sala de aula, ou fora dela, sempre tendo como objetivo o desenvolvimento das estruturas cognitivas no que tange a racionalização de problemas.

\section{Considerações finais}

O Pensamento Computacional constitui uma poderosa ferramenta teórica para solução de problemas e tem sido cada vez mais investigado no meio científico e aplicado educacionalmente. O trabalho apresentado demonstrou como os 04 (quatro) pilares do Pensamento Computacional (PC) foram aplicados à mecânica de um jogo de propósito educacional que pretende trabalhar com o tratamento de lixo em uma perspectiva de busca pela sustentabilidade, demonstrando com isso que, conforme a afirmação de Wing (2017), 
VII Congresso Brasileiro de Informática na Educação (CBIE 2018)

Anais dos Workshops do VII Congresso Brasileiro de Informática na Educação (WCBIE 2018)

PC pode sim permear diversas áreas do conhecimento e não somente a Ciência da Computação.

Os play-tests iniciais reforçam a afirmação da literatura de que jogos desenvolvidos sobre a perspectiva do Pensamento Computacional podem proporcionar aos usuários uma experiência dinâmica, motivadora e desafiadora e, quando atrelados a assuntos ou conhecimentos de cunho social como a sustentabilidade, possibilitam que o tema seja visto de maneira mais lúdica, o que promove uma aprendizagem mais eficiente.

Como trabalhos em andamento, estão sendo acrescentadas novas fases ao jogo e inseridos mais elementos de recompensa, como easter eggs, de tal forma a se criar um gameplay mais divertido e manter o jogador imerso, outra mudança em processo de desenvolvimento é o design do jogo, a nova versão tem estilo “material design” pela popularidade de aplicativos semelhantes indicados para a faixa etária do jogo.

\section{Referências}

Alves, F. (2015). Gamification: Como criar experiências de aprendizagem engajadoras: DVS Editora.

BBC (2018). Introduction to computational thinking. 2018, from https://www.bbc.com/bitesize/guides/zp92mp3/revision/1

Berg Marklund, B. (2013). Games in formal educational settings: Obstacles for the development and use of learning games. University of Skövde.

Dias, J., Mekaro, M., Lu, J., Sorrentino, G., Otsuka, J., Beder, D., . . Fonseca, L. M. M. (2015). Desenvolvimento e avaliação de um jogo educacional digital para enfrentamento da obesidade infantil. Paper presented at the Anais dos Workshops do Congresso Brasileiro de Informática na Educação.

Dichev, C., \& Dicheva, D. (2017). Gamifying education: what is known, what is believed and what remains uncertain: a critical review. International Journal of Educational Technology in Higher Education, 14(1), 9.

Farias, A., Andrade, W., \& Alencar, R. (2015). Pensamento computacional em sala de aula: Desafios, possibilidades e a formaçao docente. Paper presented at the Anais dos Workshops do Congresso Brasileiro de Informática na Educação.

Fogel, R. W. (1994). Economic growth, population theory, and physiology: the bearing of long-term processes on the making of economic policy: National Bureau of Economic Research.

Fu, F.-L., Su, R.-C., \& Yu, S.-C. (2009). EGameFlow: A scale to measure learners' enjoyment of e-learning games. Computers \& Education, 52(1), 101-112.

Gros, B. (2003). The impact of digital games in education. First Monday, 8(7), 6-26.

Hsiao, H.-C. (2007). A brief review of digital games and learning. Paper presented at the Digital Game and Intelligent Toy Enhanced Learning, 2007. DIGITEL'07. The First IEEE International Workshop on.

Jacobi, P. R. (2003). Educação ambiental, cidadania e sustentabilidade. Cadernos de pesquisa(118), 189-205. 
VII Congresso Brasileiro de Informática na Educação (CBIE 2018)

Anais dos Workshops do VII Congresso Brasileiro de Informática na Educação (WCBIE 2018)

Mayer, R. E. (2015). On the need for research evidence to guide the design of computer games for learning. Educational psychologist, 50(4), 349-353.

McFarlane, A., Sparrowhawk, A., \& Heald, Y. (2002). Report on the educational use of games: TEEM (Teachers evaluating educational multimedia), Cambridge.

Meadows, D., \& Randers, J. (2012). The limits to growth: the 30-year update: Routledge.

Mitchell, A., \& Savill-Smith, C. (2004). The use of computer and video games for learning: A review of the literature.

Qian, M., \& Clark, K. R. J. C. i. H. B. (2016). Game-based Learning and 21st century skills: A review of recent research. 63, 50-58.

Sachs, I. (1989). Desarrollo sustentable, bio-industrialización descentralizada y nuevas configuraciones rural-urbanas. El caso de la India y el Brasil. Pensamiento Iberoamericano, 16, 235-256.

Sauvé, L. (2005). Educação Ambiental: possibilidades e limitações.

Savi, R., \& Ulbricht, V. R. (2008). Jogos digitais educacionais: benefícios e desafios. RENOTE, 6(1).

Siche, R., Agostinho, F., Ortega, E., \& Romeiro, A. (2007). Índices versus indicadores: precisões conceituais na discussão da sustentabilidade de países. Ambiente \& sociedade.

Silva, A. A., \& Passerino, L. M. J. R. r. n. t. n. e. P. A., RS. (2007). A Fazenda: software educativo para a educação ambiental.

Smith, N., Sutcliffe, C., \& Sandvik, L. (2014). Code club: bringing programming to UK primary schools through scratch. Paper presented at the Proceedings of the 45th ACM technical symposium on Computer science education.

Tsuda, M., Sanches, V. M., Ferreira, T. G., Otsuka, J. L., \& Beder, D. M. (2014). Análise de métodos de avaliação de jogos educacionais. Proceedings of XIII SBGames, 12-14.

Wing, J. M. (2006). Computational thinking. Communications of the ACM, 49(3), 33-35.

Wing, J. M. (2017). Computational thinking's influence on research and education for all. Italian Journal of Educational Technology, 25(2), 7-14. 